\title{
Synchronization-Based Guaranteed-Performance Formation Design for Swarm Systems
}

\author{
Hongtao Dang, ${ }^{1}$ Yichen Du, ${ }^{1}$ Lingyun Kong, ${ }^{1}$ Hui Yao, ${ }^{2}$ and Jianxiang Xi $\mathbb{D}^{2}$ \\ ${ }^{1}$ Shaanxi Engineering Research Center of Controllable Neutron Source, School of Science, Xijing University, Xi'an 710123, China \\ ${ }^{2}$ Rocket Force University of Engineering, Xi'an 710025, China \\ Correspondence should be addressed to Jianxiang Xi; xijx07@mails.tsinghua.edu.cn
}

Received 9 May 2020; Revised 17 August 2020; Accepted 28 August 2020; Published 9 September 2020

Academic Editor: Carlos Aguilar-Ibanez

Copyright $(0) 2020$ Hongtao Dang et al. This is an open access article distributed under the Creative Commons Attribution License, which permits unrestricted use, distribution, and reproduction in any medium, provided the original work is properly cited.

Guaranteed-performance formation control for swarm systems with the second-order dynamics is investigated based on the synchronization control strategy. Firstly, a new formation protocol is presented, where the weights of connected edges are adaptively regulated and the performance constraint is imposed. Then, on the basis of the Riccati inequality, sufficient conditions for synchronization-based guaranteed-performance formation are proposed, and an explicit expression of the guaranteedperformance cost is shown, where it is fully distributed to design gain matrices of the formation protocol in the sense that it is independent of global information of swarm systems. Moreover, the whole motion of a swarm system is determined, which is associated with initial states of all agents and formation control vectors. Finally, two numerical examples are shown to demonstrate theoretical conclusions, where the static whole motion and the dynamic whole motion are considered, respectively.

\section{Introduction}

Recently, a lot of researchers paid their attention to distributed coordinated control of swarm systems since they have wide foregrounds in many fields, such as animal flocking [1, 2], network synchronization [3-8], and formation of multiple intelligent agents [9-13]. Distributed formation originates from the animal swarm motions with local communication interactions, and it is needed that many intelligent agents construct some structure and operate with this structure.

In [14], Ren summarized and commented on the existing formation approaches containing the virtual-structure approach, the behavior-based approach, and the synchronization-based method. It was pointed out that the virtualstructure approach is centralized, and the behavior-based approach cannot be analyzed by establishing accurate models and implementing rigorous deduction although it is distributed. The synchronization-based formation control approach is valid to realize specific structures by designing formation control vectors, where some coordinated variables in practical swarm systems are chosen and these errors between coordinated variables and formation control vectors are required to achieve synchronization. The synchronization-based formation control approach can overcome some drawbacks of the virtual-structure approach and the behavior-based approach, and it can establish formation in a distributed mode.

On the basis of the synchronization-based formation control approach, the following several important works were finished. In [15], formation stability was discussed by the Nyquist criterion and a necessary and sufficient condition was proposed. Du et al. [16] realized the multiple intelligent robot formation by a new nonlinear control protocol, where formation can be achieved in a finite time. In [17-26], the impacts of some practical factors including time delays, intermittent communications, and external disturbances on formation control and its practical applications, were well dealt with. Xiao et al. [27] constructed nonlinear control protocols to realize finite-time formation for swarm systems with each agent modeled by a first-order integrator. In [28], formation control for swarm systems with each agent modeled as a second-order integrator was addressed, where the formation experiment of multiple quadrotors was 
finished and the communication network has a leader-following structure. Dong and $\mathrm{Hu}$ [29] investigated formation tracking for swarm systems with multiple leaders and sufficient conditions for formation tracking were presented.

In [15-29], the formation regulation performance was not considered, which can be modeled as global suboptimal or optimal formation design problems. Inspired by the guaranteed-cost synchronization control for swarm systems addressed in [30], Wang et al. [31] investigated guaranteedperformance formation control with limited communication. Yu et al. [32] discussed guaranteed-performance formation control for swarm systems with time-varying delays and unknown disturbances and presented the formation control criteria on the basis of the linear matrix inequality, where no approach was proposed to determine the specific structure of the guaranteed-performance cost. Moreover, the associated formation control criteria in [31, 32] are associated with the global information of swarm systems, such as the Laplacian matrix of the communication network or its nonzero eigenvalues; that is, those criteria are not fully distributed since gain matrices may be changed when some agents are removed or some agents are added in swarm systems. As far as we know, the synchronization-based guaranteed-performance formation design problem for swarm systems with the fully distributed control strategy is still not comprehensively studied.

This paper investigates a fully distributed strategy to realize synchronization-based guaranteed-performance formation design for swarm systems. A formation protocol is proposed on the basis of state errors and formation errors among adjacent agents, which contains the guaranteedperformance constraint and the regulation mechanism of edge weights. Furthermore, by constructing Lyapunov functions with the adaptively regulated weight terms, the translation adaptive method is proposed to eliminate the impacts of nonzero eigenvalues, and sufficient conditions for synchronization-based guaranteed-performance formation are proposed, which are fully distributed since they are not associated with global information of swarm systems. Moreover, an explicit expression of the guaranteed-performance cost is shown, and the whole motion of a swarm system is determined, which may be static and can also move in the uniform linear mode according to different initial values of velocity-similar states of all agents.

Compared with the closely associated literatures on formation control, our work has three critical characteristics. Firstly, synchronization-based guaranteed-performance formation criteria are fully distributed; that is, the gain matrix design is independent of the global information of communication networks of swarm systems. In [31, 32], the formation performance among neighboring agents can only be regulated. Thirdly, the whole motion of a swarm system depends on the average of initial values of velocity-similar states and position-similar states and formation control vectors. The whole motion of a swarm system was not determined in [32], and the approach in [31] is no longer valid due to adaptively regulated edge weights.

The remainder of this paper is arranged as follows. In Section 2, the problem formulation is stated on the basis of the graph theory and the neighboring relationship, and a formation protocol with adaptively regulated weights and the performance constraint is shown. Section 3 proposes sufficient conditions for synchronization-based guaranteedperformance formation control according to the Riccati inequality and determines an explicit expression of the guaranteed-performance cost. Furthermore, the motion of a swarm system as a whole is determined. In Section 4, two numerical examples are given to demonstrate theoretical results. In Section 5, conclusion comments are provided.

1.1. Notations. $\mathbb{R}^{d}$ and $\mathbb{R}^{d \times d}$ are applied to represent the $d$ and $d \times d$-dimensional real column vector space and real matrix space, respectively. 0 is used to stand for the zero matrix with all components zero and the zero number as a special case. The notation $I_{N}$ represents the $N$-dimensional identity matrix, and $\mathbf{1}_{N}$ is used to denote an $N$-dimension column vector with all components 1 . The notation $\otimes$ denotes the Kronecker product. The symbols $Q^{\mathrm{T}}=Q<0$ and $Q^{T}=Q>0$ mean that the symmetric matrix $Q$ is negative definite and positive definite, respectively, where $Q^{T}$ denotes the transpose of the matrix $Q$.

\section{Problem Formulation}

2.1. Modeling Communication Structure. The communication structure of a swarm system can be depicted by an undirected network $G=\mathscr{G}(\mathscr{V}, \mathscr{E})$, where $\mathscr{V}=\left\{V_{1}, V_{2}, \ldots, V_{N}\right\} \quad$ is the node set and $\mathscr{E} \subseteq\left\{\left(V_{i}, V_{j}\right), \quad i \neq j, V_{i}, V_{j} \in \mathscr{V}\right\}$ is the edge set. It is assumed that the communication network $G$ is connected. Each agent in this swarm system is denoted by a certain node in the communication network, and each communication channel of this swarm system is described by some edge in the communication network. The notation $\mathcal{N}_{i}$ is used to represent the neighbor set of the $i$ th agent, which contains all agents connecting with agent $i$ and is not null since the communication network $G$ is connected.

The weighted matrix of the communication network $G$ is denoted by a symmetric matrix $W(t)=\left[l_{i j} w_{i j}(t)\right]_{N \times N}$ called the adjacency matrix, where $l_{i i}=0, l_{i j}=1$ if $j$ is a neighbor of agent $i, l_{i j}=0$ if $j$ is not a neighbor of agent $i$, and $w_{i j}(t)>0$ is the time-varying communication weight between agents $j$ and $i$. When $l_{i j}=0, w_{i j}(t)$ stands for a virtual time-varying communication weight between agents $j$ and $i$. The matrix $D(t)=\operatorname{diag}\left\{d_{1}(t), d_{2}(t), \ldots, d_{N}(t)\right\}$ with $d_{i}(t)=\sum_{j=1, j \neq i}^{N}$ $l_{i j} w_{i j}(t)$ represents the in-degree matrix of the communication network $G$. Let $L_{w(t)}=D(t)-W(t)$ denote the Laplacian matrix of the communication network $G$, which is time-varying.

More basic concepts on graph theory can be found in [33]. The following lemma is required to obtain our main conclusions, which shows some basic properties of the Laplacian matrix.

Lemma 1 (see [33]). The Laplacian matrix $L_{w(t)}$ at least owns a zero eigenvalue with the associated eigenvector $\mathbf{1}_{N}$. If the communication network $G$ is connected, then the zero eigenvalue is simple and all the other $N-1$ ones are positive. 
2.2. Synchronization-Based Guaranteed-Performance Formation. Consider a swarm system with $N$ second-order agents with the same dynamics, and each agent is modeled as

$$
\left\{\begin{array}{l}
\dot{x}_{i}(t)=v_{i}(t), \\
\dot{v}_{i}(t)=u_{i}(t),
\end{array}\right.
$$

where $i \in\{1,2, \ldots, N\}, x_{i}(t) \in \mathbb{R}^{d}$ is the position-similar state, $v_{i}(t) \in \mathbb{R}^{d}$ is the velocity-similar state, and $u_{i}(t) \in \mathbb{R}^{d}$ is the control input determining by the formation protocol, respectively. The given vector $\iota=\left[\iota_{1}^{\mathrm{T}}, \iota_{2}^{\mathrm{T}}, \ldots, \iota_{N}^{\mathrm{T}}\right]^{\mathrm{T}}$ with $\iota_{i} \in \mathbb{R}^{d}$ is used to determine specific formation structure, which is called a formation control vector. Now, we show the definition of synchronization-based formation.

Definition 1. Swarm system (1) is said to achieve synchronization-based formation if $\lim _{t \rightarrow \infty}\left(x_{i}(t)-x_{j}(t)-t_{i}+\right.$ $\left.\iota_{j}\right)=0$ and $\lim _{t \rightarrow \infty}\left(v_{i}(t)-v_{j}(t)\right)=0$ for $\forall i, j \in\{1,2, \ldots, N\}$.

Let $\delta_{i j}(t)=\left[\delta \varepsilon_{i j}^{\mathrm{T}}(t), \delta v_{i j}^{\mathrm{T}}(t)\right]^{\mathrm{T}}$, where $\delta \varepsilon_{i j}(t)=\varepsilon_{j}(t)-$ $\varepsilon_{i}(t)$ with $\varepsilon_{i}(t)=x_{i}(t)-t_{i}$ and $\delta v_{i j}(t)=v_{j}(t)-v_{i}(t)$. Due to $\dot{\varepsilon}_{i}(t)=\dot{x}_{i}(t)=v_{i}(t)$, one can see by (1) that

$$
\left\{\begin{array}{l}
\dot{\varepsilon}_{i}(t)=v_{i}(t), \\
\dot{v}_{i}(t)=u_{i}(t) .
\end{array}\right.
$$

Thus, $\lim _{t \rightarrow \infty}\left(\varepsilon_{i}(t)-\varepsilon_{j}(t)\right)=0$ can guarantee that $\lim _{t \rightarrow \infty}\left(x_{i}(t)-x_{j}(t)-t_{i}+\iota_{j}\right)=0$, which means that the formation control problem is transformed into the synchronization one by (2). Here, we summarize a lemma as follows.

Lemma 2. Swarm system (1) achieves synchronization-based formation if $\lim _{t \rightarrow \infty}\left(\varepsilon_{i}(t)-\varepsilon_{j}(t)\right)=0$ and $\lim _{t \rightarrow \infty}\left(v_{i}(t)-\right.$ $\left.v_{j}(t)\right)=0$ for $\forall i, j \in\{1,2, \ldots, N\}$.

Swarm system (1) contains two state components; that is, a position-similar component and a velocity-similar component. On the basis of this fact, the control input $u_{i}(t)$ is constructed as

$$
u_{i}(t)=u_{i \varepsilon}(t)+u_{i v}(t)
$$

where

$$
\begin{aligned}
& u_{i \varepsilon}(t)=K_{u 1} \sum_{j \in \mathcal{N}_{i}} w_{i j}(t)\left(\varepsilon_{j}(t)-\varepsilon_{i}(t)\right), \\
& u_{i v}(t)=K_{u 2} \sum_{j \in \mathcal{N}_{i}} w_{i j}(t)\left(v_{j}(t)-v_{i}(t)\right) .
\end{aligned}
$$

And $K_{u 1}$ and $K_{u 2}$ are gain matrices with compatible dimensions. Let $\delta_{i j}(t)=\left[\delta \varepsilon_{i j}^{\mathrm{T}}(t), \delta v_{i j}^{\mathrm{T}}(t)\right]^{\mathrm{T}} \quad$ with $\delta \varepsilon_{i j}(t)=\varepsilon_{j}(t)-\varepsilon_{i}(t)$ and $\delta v_{i j}(t)=v_{j}(t)-v_{i}(t) \quad$ and $K_{u}=\left[K_{u 1}, K_{u 2}\right]$; then, it can be found by (3) that

$$
u_{i}(t)=K_{u} \sum_{j \in \mathcal{N}_{i}} w_{i j}(t) \delta_{i j}(t)
$$

Thus, we propose the following guaranteed-performance formation protocol:

$$
\left\{\begin{array}{l}
u_{i}(t)=K_{u} \sum_{j \in \mathcal{N}_{i}} w_{i j}(t) \delta_{i j}(t), \\
\dot{w}_{i j}(t)=\delta_{i j}^{\mathrm{T}}(t) K_{w} \delta_{i j}(t), \\
J_{r}=\frac{1}{N} \sum_{i=1}^{N} \sum_{j=1}^{N} \int_{0}^{\infty} \delta_{i j}^{\mathrm{T}}(t) Q \delta_{i j}(t) \mathrm{d} t,
\end{array}\right.
$$

where $i=1,2, \ldots, N, Q^{\mathrm{T}}=Q>0$, and $K_{w}^{\mathrm{T}}=K_{w}>0$ is a gain matrix. One can find that the derivative of the communication weight $w_{i j}(t)$ is nonnegative, so the communication weight $w_{i j}(t)$ is adaptively adjusted and positive. Particularly, it may be consistently increasing until agents $i$ and $j$ achieve the desired formation. In this case, the upper bound of $w_{i j}(t)$ is set as $\gamma_{i j}$. Here, we give the definition of synchronization-based guaranteed-performance formation control.

Definition 2. Swarm system (1) is said to be synchronizationbased guaranteed-performance formation achievable by formation protocol (6) if there exist $J_{r}^{*}>0, K_{u}$, and $K_{w}$ such that $J_{r} \leq J_{r}^{*}, \lim _{t \rightarrow \infty}\left(x_{i}(t)-x_{j}(t)-\iota_{i}+\iota_{j}\right)=0$ and $\lim _{t \rightarrow \infty}\left(v_{i}(t)-v_{j}(t)\right)=0$ for $\forall i, j \in\{1,2, \ldots, N\}$.

The main objective of this paper is to design gain matrices $K_{u}$ and $K_{w}$ such that swarm system (1) with formation protocol (6) achieves synchronization-based guaranteedperformance formation and to determine an explicit expression of the guaranteed-performance cost $J_{r}^{*}$. Moreover, the whole motion of swarm system (1) is determined when synchronization-based guaranteed-performance formation is achieved.

Remark 1. Formation protocol (6) owns two important features. The first one is that the neighbor set of all agents is time-invariant, but the communication weight $w_{i j}(t)(i \neq j)$ is not, which is time-varying and can be continuously increasing if the formation error $\delta_{i j}(t)(i, j \in\{1,2, \ldots, N\})$ between agents $i$ and $j$ is not zero. Particularly, the increasing value $w_{i j}(t)(i \neq j)$ becomes more larger when the formation error $\delta_{i j}(t)(i, j \in\{1,2, \ldots, N\})$ between agents $i$ and $j$ becomes big; that is, more control impacts are imposed on these two agents to make them achieve the desired formation more quickly. In this case, this protocol can also be called the adaptive formation protocol. The second one is that formation protocol (6) ensures the formation regulation performance among all agents by the quadratic index function $J_{r}$ although some agents may be unconnected, which indicates that the whole formation performance of swarm system (1) can be regulated by formation protocol (6).

\section{Synchronization-Based Guaranteed- Performance Formation}

In this section, firstly, by the time-invariant nonsingular transformation, the formation control problems are transformed into asymptotic stability ones. Then, sufficient conditions for synchronization-based guaranteed- 
performance formation are proposed on the basis of the Riccati inequality. Finally, an approach is shown to determine the whole motion of a swarm system when synchronization-based guaranteed-performance formation is achieved.

Let $\chi_{i}^{\mathrm{T}}(t)=\left[x_{i}^{\mathrm{T}}(t), v_{i}^{\mathrm{T}}(t)\right]^{\mathrm{T}}(i=1,2, \ldots, N), \chi(t)=\left[\chi_{1}^{\mathrm{T}}\right.$ $\left.(t), \chi_{2}^{\mathrm{T}}(t), \ldots, \chi_{N}^{\mathrm{T}}(t)\right]^{\mathrm{T}}, \quad$ and $u(t)=\left[u_{1}^{\mathrm{T}}(t), u_{2}^{\mathrm{T}}(t), \ldots, u_{N}^{\mathrm{T}}\right.$ $(t)]^{\mathrm{T}}$; then, swarm system (1) can be rewritten in a compact form as

$$
\dot{\chi}(t)=\left(I_{N} \otimes A\right) \chi(t)+\left(I_{N} \otimes B\right) u(t),
$$

where

$$
\begin{aligned}
& A=\left[\begin{array}{ll}
0 & I_{d} \\
0 & 0
\end{array}\right], \\
& B=\left[\begin{array}{c}
0 \\
I_{d}
\end{array}\right] .
\end{aligned}
$$

One can set that $l=\left[l_{1}^{\mathrm{T}}, l_{2}^{\mathrm{T}}, \ldots, l_{N}^{\mathrm{T}}\right]^{\mathrm{T}}$ with $l_{i}=\left[t_{i}^{\mathrm{T}}, 0\right]^{\mathrm{T}}$ and $\omega(t)=\left[\omega_{1}^{\mathrm{T}}(t), \omega_{2}^{\mathrm{T}}(t), \ldots, \omega_{N}^{\mathrm{T}}(t)^{\mathrm{T}}\right]$, with $\omega_{i}(t)=\left[\chi_{i}^{\mathrm{T}}(t)-l_{i}^{\mathrm{T}}\right]^{\mathrm{T}}$. Thus, one can see that $\omega(t)=\chi(t)-l$, which can be used to write the whole dynamics into an impact form.

Because the formation vector $l$ is constant, one can see that $\dot{l}=0$. Due to $\omega(t)=\chi(t)-l$, from (6) and (7), one can obtain that

$$
\dot{\omega}(t)=\left(I_{N} \otimes A-L_{w(t)} \otimes B K_{u}\right) \omega(t)+\left(I_{N} \otimes A\right) l,
$$

where $L_{w(t)}$ is the time-varying Laplacian matrix of the communication network $G$. Due to

$$
A l_{i}=\left[\begin{array}{cc}
0 & I_{d} \\
0 & 0
\end{array}\right]\left[\begin{array}{l}
\iota_{i} \\
0
\end{array}\right]=\left[\begin{array}{l}
0 \\
0
\end{array}\right],
$$

where $i=1,2, \ldots, N$, one can derive that

$$
\left(I_{N} \otimes A\right) l=0 .
$$

Thus, one can show by (9) and (11) that

$$
\dot{\omega}(t)=\left(I_{N} \otimes A-L_{w(t)} \otimes B K_{u}\right) \omega(t) .
$$

From Lemma 1, one can find that $L_{w(t)} \mathbf{1}_{N}=0$. Let $0=$ $\lambda_{1}<\lambda_{2} \leq \cdots \leq \lambda_{N}$ denote the eigenvalues of $L_{w(0)}$. Because the communication network $G$ owns the connected property, one can find a constant nonsingular matrix $U=\left[\mathbf{1}_{N} / \sqrt{N}, \bar{U}\right]$ with $U^{\mathrm{T}} U=I_{N}$ such that

$$
U^{\mathrm{T}} L_{w(t)} U=\left[\begin{array}{cc}
0 & 0 \\
0 & \bar{U}^{\mathrm{T}} L_{w(t)} \bar{U}
\end{array}\right],
$$

where $\quad \bar{U}^{\mathrm{T}} L_{w(0)} \bar{U}=\operatorname{diag}\left\{\lambda_{2}, \lambda_{3}, \ldots, \lambda_{N}\right\}$. Let $\kappa(t)=$ $\left(U^{\mathrm{T}} \otimes I_{2 d}\right) \omega(t)=\left[\kappa_{1}^{\mathrm{T}}(t), \kappa_{e}^{\mathrm{T}}(t)\right]^{\mathrm{T}}$, where $\kappa_{1}(t) \in \mathbb{R}^{2 d}$ and $\kappa_{e}(t)=\left[\kappa_{2}^{\mathrm{T}}(t), \kappa_{3}^{\mathrm{T}}(t), \ldots, \kappa_{N}^{\mathrm{T}}(t)\right]^{\mathrm{T}} \in \mathbb{R}^{2 d(N-1)} \quad$ with $\kappa_{i}(t) \in \mathbb{R}^{2 d}(i=2,3, \ldots, N)$; then swarm system (12) can be transformed by (13) into two independent parts.

$$
\begin{aligned}
& \dot{\kappa}_{1}(t)=A \kappa_{1}(t), \\
& \dot{\kappa}_{e}(t)=\left(I_{N-1} \otimes A-\bar{U}^{\mathrm{T}} L_{w(t)} \bar{U} \otimes B K_{u}\right) \kappa_{e}(t) .
\end{aligned}
$$

One can set that

$$
\begin{aligned}
& \widetilde{\kappa}_{1}(t)=U e_{1} \otimes \kappa_{1}(t)=\frac{1}{\sqrt{N}} 1_{N} \otimes \kappa_{1}(t), \\
& \widetilde{\kappa}_{e}(t)=\sum_{i=2}^{N} U e_{i} \otimes \kappa_{i}(t),
\end{aligned}
$$

where the $N$-dimensional column vector $e_{i}(i \in\{1,2, \ldots, N\})$ owns the property that the $i$ th element is 1 and 0 elsewhere. One can find by (16) and (17) that

$$
\begin{aligned}
& \widetilde{\kappa}_{1}(t)=\left(U \otimes I_{2 d}\right)\left[\kappa_{1}^{\mathrm{T}}(t), 0^{\mathrm{T}}\right]^{\mathrm{T}}, \\
& \widetilde{\kappa}_{e}(t)=\left(U \otimes I_{2 d}\right)\left[0^{\mathrm{T}}, \kappa_{e}^{\mathrm{T}}(t)\right]^{\mathrm{T}} .
\end{aligned}
$$

Because $U \otimes I_{2 d}$ is invertible, it can be found that these two vectors $\widetilde{\kappa}_{1}(t)$ and $\widetilde{\kappa}_{e}(t)$ are linearly independent. Due to

$$
\begin{aligned}
& {\left[\kappa_{1}^{\mathrm{T}}(t), \kappa_{e}^{\mathrm{T}}(t)\right]^{\mathrm{T}}=\left(U^{\mathrm{T}} \otimes I_{2 d}\right)\left(\widetilde{\kappa}_{1}(t)+\widetilde{\kappa}_{e}(t)\right),} \\
& {\left[\kappa_{1}^{\mathrm{T}}(t), \kappa_{e}^{\mathrm{T}}(t)\right]^{\mathrm{T}}=\left(U^{\mathrm{T}} \otimes I_{2 d}\right) \omega(t),}
\end{aligned}
$$

one has $\omega(t)=\widetilde{\kappa}_{1}(t)+\widetilde{\kappa}_{e}(t)$. If $\lim _{t \rightarrow \infty} \widetilde{\kappa}_{e}(t)=0$, then $\lim _{t \rightarrow \infty}\left(\omega(t)-\widetilde{\kappa}_{1}(t)\right)=0$. From (16), $\lim _{t \rightarrow \infty} \widetilde{\kappa}_{e}(t)=0$ is equivalent to $\lim _{t \rightarrow \infty} \kappa_{e}(t)=0$ since the transformation matrix $U \otimes I_{2 d}$ is nonsingular. Thus, by Definition 1 and Lemma 2, we can obtain the following lemma.

Lemma 3. Swarm system (1) achieves synchronization-based formation if and only if $\lim _{t \rightarrow \infty} \kappa_{e}(t)=0$.

The following theorem proposes an approach to determine gain matrices $K_{u}$ and $K_{w}$ such that swarm system (1) with formation protocol (6) achieves synchronization-based guaranteed-performance formation, which is independent of the eigenvalues of the time-varying Laplacian matrix of the communication network.

Theorem 1. For any given $\gamma>0$, swarm system (1) is synchronization-based guaranteed-performance formation achievable by formation protocol (6) with $K_{u}=B^{T} P$ and $K_{w}=P B B^{T} P$ if there exists a symmetric and positive definite matrix $P$ such that $P A+A^{T} P-\gamma P B B^{T} P+2 Q \leq 0$. In this case, an explicit expression of the guaranteed-performance cost is

$$
J_{r}^{*}=\omega^{\mathrm{T}}(0)\left(\left(I_{N}-\frac{1}{N} \mathbf{1}_{N} \mathbf{1}_{N}^{\mathrm{T}}\right) \otimes P\right) \omega(0)+\gamma \int_{0}^{+\infty} \omega^{\mathrm{T}}(t)\left(\left(I_{N}-\frac{1}{N} \mathbf{1}_{N} \mathbf{1}_{N}^{\mathrm{T}}\right) \otimes P B B^{\mathrm{T}} P\right) \omega(t) \mathrm{d} t .
$$


Proof. Construct the following Lyapunov functional candidate:

$$
V(t)=\kappa_{e}^{\mathrm{T}}(t)\left(I_{N-1} \otimes P\right) \kappa_{e}(t)+\sum_{i=1}^{N} \sum_{j \in \mathcal{N}_{i}} \frac{\left(w_{i j}(t)-w_{i j}(0)\right)^{2}}{2}+\frac{\gamma}{2 N} \sum_{i=1}^{N} \sum_{j=1, j \neq i}^{N}\left(\gamma_{i j}-w_{i j}(t)\right) .
$$

According to $P^{\mathrm{T}}=P>0$ and $\gamma_{i j} \geq w_{i j}(t)$, one can find that $V(t) \geq 0$. Because the communication network $G$ is undirected, it can be found that the time-varying Laplacian matrix $L_{w(t)}$ is symmetric. Let $K_{u}=B^{T} P$, then, according to (15) and (20), one can deduce that

$$
\dot{V}(t)=\kappa_{e}^{\mathrm{T}}(t)\left(I_{N-1} \otimes\left(P A+A^{\mathrm{T}} P\right)\right) \kappa_{e}(t)-\kappa_{e}^{\mathrm{T}}(t)\left(2 \bar{U}^{\mathrm{T}} L_{w(t)} \bar{U} \otimes P B B^{\mathrm{T}} P\right) \kappa_{e}(t)+\sum_{i=1}^{N} \sum_{j \in \mathcal{N}_{i}}\left(w_{i j}(t)-w_{i j}(0)\right) \dot{w}_{i j}(t)-\frac{\gamma}{2 N} \sum_{i=1}^{N} \sum_{j=1, j \neq i}^{N} \dot{w}_{i j}(t) .
$$

Because the time-invariant transformation matrix $U$ is orthonormal, one can see that $\overline{U U}^{\mathrm{T}}=L_{N}$, where $L_{N}$ stands for the Laplacian matrix of a communication network $G^{*}$ and all its edge weight is $1 / N$; that is,

$$
L_{N}=I_{N}-\frac{1}{N} 1_{N} 1_{N}^{\mathrm{T}}
$$

By (6) and (13), it can be shown that

$$
\begin{aligned}
& \sum_{i=1}^{N} \sum_{j \in \mathcal{N}_{i}}\left(w_{i j}(t)-w_{i j}(0)\right) \dot{w}_{i j}(t)-\frac{\gamma}{2 N} \sum_{i=1}^{N} \sum_{j=1, j \neq i}^{N} \dot{w}_{i j}(t) \\
& =\kappa_{e}^{\mathrm{T}}(t)\left(\left(2 \bar{U}^{\mathrm{T}} L_{w(t)} \bar{U}-2 \bar{U}^{\mathrm{T}} L_{w(0)} \bar{U}-\gamma I_{N-1}\right) \otimes K_{w}\right) \kappa_{e}(t) .
\end{aligned}
$$
that

$$
\dot{V}(t)=\kappa_{e}^{T}(t)\left(I_{N-1} \otimes\left(P A+A^{T} P\right)-\left(2 \bar{U}^{T} L_{w(0)} \bar{U}+\gamma I_{N-1}\right) \otimes P B B^{T} P\right) \kappa_{e}(t) .
$$

Due to $\bar{U}^{\mathrm{T}} L_{w(0)} \bar{U}=\operatorname{diag}\left\{\lambda_{2}, \lambda_{3}, \ldots, \lambda_{N}\right\} \quad$ with $\lambda_{2} \leq \lambda_{3} \leq \cdots \leq \lambda_{N}$ being the nonzero eigenvalues of $L_{w(0)}$, it can be found that

$$
\dot{V}(t) \leq \sum_{i=2}^{N} \kappa_{i}^{\mathrm{T}}(t)\left(P A+A^{\mathrm{T}} P-\left(2 \lambda_{i}+\gamma\right) P B B^{\mathrm{T}} P\right) \kappa_{i}(t) .
$$

One can see that $P A+A^{\mathrm{T}} P-\gamma P B B^{\mathrm{T}} P<0 \quad$ if $P A+A^{\mathrm{T}} P-\gamma P B B^{\mathrm{T}} P+2 Q \leq 0$. In this case, it can be obtained that $V(t) \leq-\varepsilon\left\|\kappa_{e}\right\|^{2}$ with some $\varepsilon>0$, which means that $\lim _{t \rightarrow \infty} \kappa_{e}(t)=0$; that is, swarm system (1) achieves synchronization-based formation by Lemma 3.

In the sequel, we discuss the impacts of the guaranteedperformance function and determine an explicit expression of the guaranteed-performance cost $J_{r}^{*}$. It can be shown that

$$
\omega^{\mathrm{T}}(t)\left(L_{N} \otimes I\right) \omega(t)=\kappa_{e}^{\mathrm{T}}(t) \kappa_{e}(t) .
$$

Due to $\overline{U U}^{\mathrm{T}}=L_{N}$, one can see that

$$
\omega^{\mathrm{T}}(t)\left(L_{N} \otimes Q\right) \omega(t)=\sum_{i=2}^{N} \kappa_{i}^{\mathrm{T}}(t) Q \kappa_{i}(t) .
$$

Define

$$
\nabla=\sum_{i=1}^{N} \sum_{j=1}^{N}\left(\omega_{j}(t)-\omega_{i}(t)\right)^{\mathrm{T}} \mathrm{Q}\left(\omega_{j}(t)-\omega_{i}(t)\right)
$$

Then, one has

$$
J_{r}^{h} \triangleq \frac{1}{N} \int_{0}^{h} \nabla \mathrm{d} t=\sum_{i=2}^{N} \int_{0}^{h} 2 \kappa_{i}^{\mathrm{T}}(t) Q \kappa_{i}(t) \mathrm{d} t,
$$

where $h$ is a scalar larger than zero and $2 Q$ is introduced in the Riccati inequality. Since $\lambda_{i}>0(i=2,3, \ldots, N)$, it can be shown by (26) that

$$
\dot{V}(t) \leq \sum_{i=2}^{N} \kappa_{i}^{\mathrm{T}}(t)\left(P A+A^{\mathrm{T}} P-\gamma P B B^{\mathrm{T}} P\right) \kappa_{i}(\mathrm{t})
$$

Due to

$$
\int_{0}^{h} V(t) \mathrm{d} t+\dot{V}(0)-V(h)=0,
$$

one can see that 


$$
J_{r}^{h}=\sum_{i=2}^{N}\left(\int_{0}^{h}\left(2 \kappa_{i}^{\mathrm{T}}(t) Q \kappa_{i}(t)+\dot{V}(t)\right) \mathrm{d} t+V(0)-V(h)\right)
$$

From (29) to (32), one can find that

$$
J_{r}^{h} \leq \sum_{i=2}^{N}\left(\int_{0}^{h} \kappa_{i}^{\mathrm{T}}(t)\left(P A+A^{T} P-\gamma P B B^{T} P+2 Q\right) \kappa_{i}(t) \mathrm{d} t+V(0)-V(h)\right) .
$$

If $P A+A^{\mathrm{T}} P-\gamma P B B^{\mathrm{T}} P+2 Q \leq 0$, then one can find by (34) that

$$
\lim _{h \longrightarrow \infty}\left(J_{r}^{h}-V(0)+V(h)\right)=0 .
$$

Due to $\lim _{h \longrightarrow \infty}\left(\gamma_{i j}-w_{i j}(h)\right)=0$, one can obtain by (21) that

$$
\lim _{h \longrightarrow+\infty} V(h)=\lim _{h \longrightarrow+\infty} \kappa_{e}^{\mathrm{T}}(h)\left(I_{N-1} \otimes P\right) \kappa_{e}(h)+\lim _{h \longrightarrow+\infty} \sum_{i=1}^{N} \sum_{j \in \mathcal{N}_{i}} \frac{\left(w_{i j}(h)-w_{i j}(0)\right)^{2}}{2}>0 .
$$

Thus, we can find by (35) that

$$
J_{r} \leq V(0)
$$

Furthermore, one can obtain that

$$
\frac{\gamma}{2 N} \sum_{i=1}^{N} \sum_{j=1, j \neq i}^{N}\left(\gamma_{i k}-w_{i k}(0)\right)=\frac{\gamma}{2 N} \sum_{i=1}^{N} \sum_{j=1, j \neq i}^{N} \int_{0}^{+\infty} \dot{w}_{i k}(t) \mathrm{d} t=\gamma \int_{0}^{+\infty} \omega^{T}(t)\left(\left(I_{N}-\frac{1}{N} \mathbf{1}_{N} \mathbf{1}_{N}^{T}\right) \otimes P B B^{T} P\right) \omega(t) \mathrm{d} t
$$

Due to

$$
\begin{aligned}
& \overline{U U}^{T}=I_{N}-\frac{1}{N} \mathbf{1}_{N} \mathbf{1}_{N}^{T}, \\
& \kappa_{e}(t)=\left[0_{2(N-1) d \times 2 d}, I_{2(N-1) d}\right]\left(U^{T} \otimes I_{2 d}\right) \omega(t),
\end{aligned}
$$

one has

$$
\kappa_{e}^{T}(0)\left(I_{N-1} \otimes P\right) \kappa_{e}(0)=\omega^{T}(0)\left(\left(I_{N}-\frac{1}{N} \mathbf{1}_{N} \mathbf{1}_{N}^{T}\right) \otimes P\right) \omega(0)
$$

From (21) and (37)-(39), one can obtain that an explicit expression of the guaranteed-performance cost is

$$
J_{r}^{*}=\omega^{T}(0)\left(\left(I_{N}-\frac{1}{N} \mathbf{1}_{N} \mathbf{1}_{N}^{T}\right) \otimes P\right) \omega(0)+\gamma \int_{0}^{+\infty} \omega^{T}(t)\left(\left(I_{N}-\frac{1}{N} \mathbf{1}_{N} \mathbf{1}_{N}^{T}\right) \otimes P B B^{T} P\right) \omega(t) \mathrm{d} t
$$

Moreover, $P A+A^{T} P-\gamma P B B^{T} P+2 Q \leq 0$ can guarantee that swarm system (1) is synchronization-based guaranteedperformance formation achievable by formation protocol (6) with $K_{u}=B^{T} P$ and $K_{w}=P B B^{T} P$. Thus, the conclusion of Theorem 1 can be obtained.

Now, we introduce a scalar $\mu>0$ such that $P \leq \mu I$ and one can find that $P B B^{T} P \leq \mu^{2} B B^{T}$ when $\lambda_{\text {max }}\left(B B^{T}\right) \leq 1$, where $\lambda_{\max }\left(B B^{T}\right)$ stands for the maximum eigenvalue of the matrix $B B^{T}$. By Schur complement lemma in [34], the following corollary can be obtained directly.

Theorem 2. For any given $\gamma>0$ and $\mu>0$, swarm system (1) is synchronization-based guaranteed-performance formation achievable by formation protocol (6) with $K_{u}=B^{T} P$ and $K_{w}=P B B^{T} P$ if $\lambda_{\max }\left(B B^{T}\right) \leq 1$ and there exist $\gamma>0$ and $\widetilde{P}^{T}=\widetilde{P} \geq \mu^{-1} I$ such that $A \widetilde{P}+\widetilde{P} A^{T}-\gamma B B^{T}+2 \widetilde{P} Q \widetilde{P}<0$. In this case, an explicit expression of the guaranteed-performance cost is

$$
J_{r}^{*}=\sum_{i=2}^{N}\left(\mu\left\|\kappa_{i}(0)\right\|^{2}+\gamma \mu^{2} \int_{0}^{\infty}\left\|B^{T} \kappa_{i}(t)\right\|^{2} \mathrm{~d} t\right)
$$

The Riccati inequality bounded by an identity matrix in Theorem 2 can be transformed into linear matrix inequality conditions by the Schur complement lemma, which can be solved by the feasp solver in LMI's toolbox.

Let $x_{w}(t)$ and $v_{w}(t)$ denote position-similar and velocity-similar components of the whole motion of swarm system (1), respectively. The following theorem determines the whole motion of swarm system (1) with formation protocol (6) when synchronization-based guaranteed-performance formation is achieved. 
Theorem 3. If swarm system (1) with formation protocol (6) achieves synchronization-based guaranteed-performance formation, then the motion of swarm system (1) as a whole satisfies that $x_{w}(t)=\bar{x}_{w}(0)+\bar{\imath}_{w}+\bar{v}_{w}(0) t \quad$ and $v_{w}(t)=\bar{v}_{w}(0), \quad$ where $\quad \bar{x}_{w}(0)=N^{-1} \sum_{i=1}^{N} x_{i}(0)$, $\bar{v}_{w}(0)=N^{-1} \sum_{i=1}^{N} v_{i}(0)$, and $\bar{\imath}_{w}=N^{-1} \sum_{i=1}^{N} \iota_{i}$.

Proof. If swarm system (1) achieves synchronization-based guaranteed-performance formation, then one can find that

$$
\lim _{t \longrightarrow \infty}\left(\omega(t)-\frac{1}{\sqrt{N}} 1_{N} \otimes \kappa_{1}(t)\right)=0 .
$$

That is,

$$
\lim _{t \rightarrow \infty}\left(\left[\begin{array}{c}
x_{i}(t)-\iota_{i} \\
v_{i}(t)
\end{array}\right]-\frac{1}{\sqrt{N}} \kappa_{1}(t)\right)=0 .
$$

Due to

$$
\kappa_{1}(0)=\left(e_{1}^{\mathrm{T}} \otimes I_{2 d}\right)\left(U^{\mathrm{T}} \otimes I_{2 d}\right) \omega(0)=\frac{1}{\sqrt{N}} \sum_{i=1}^{N} \omega_{i}(0),
$$

one can obtain by (14) that

$$
\kappa_{1}(t)=e^{A t} \kappa_{1}(0)=(I+A t) \kappa_{1}(0) .
$$

From (44) to (46), it can be shown that

$$
\kappa_{1}(t)=\frac{1}{N} \sum_{i=1}^{N}\left[\begin{array}{c}
x_{i}(0)-\iota_{i} \\
v_{i}(0)
\end{array}\right]+\frac{1}{N} \sum_{i=1}^{N}\left[\begin{array}{c}
v_{i}(0) \\
0
\end{array}\right] t .
$$

Thus, the conclusion of Theorem 3 can be obtained.

Remark 2. In [31, 32], sufficient conditions for formation control depend on the global information of swarm systems, such as the Laplacian matrix and its nonzero eigenvalues, etc. In those cases, formation control strategies are not fully distributed. It should be pointed out that the dilation adaptive method can realize fully distributed control as shown in [35], but it is no longer valid when the guaranteed performance is considered and becomes an important constraint for formation design. In the proof of Theorem 1, we introduce a translation scalar to eliminate the impacts of nonzero eigenvalues on guaranteed-performance formation control and realize the fully distributed control in the sense that gain matrices of the formation protocol are not associated with the global information of swarm systems. Particularly, the translation adaptive method is closely associated with the undirected property of the communication network, which introduces the Laplacian matrix of a complete graph.

Remark 3. Theorem 3 shows an approach to determine the motion of swarm system (1) with formation protocol (6) as a whole, where $\bar{x}_{w}(0)$ and $\bar{v}_{w}(0)$ are the average of initial values of position-similar states and velocity-similar states, respectively, and $\bar{l}_{w}$ is the average value of all formation control vectors. Two important aspects should be emphasized. The first one is that the formation control vectors not only determine formation structures, but also impact the whole motion. The second one is that if the average of initial values of velocity-similar states is equal to zero, then the whole formation is static as the synchronization-based guaranteed-performance formation is achieved. In this case, the center point of the whole formation is jointly determined by the average of initial values of position-similar states and the formation control vectors. However, if the average of initial values of velocity-similar states is nonzero, then the whole formation moves in the uniform linear mode when the synchronization-based guaranteed-performance formation is achieved.

\section{Numerical Simulations}

In this section, two numerical examples are provided to demonstrate the theoretical conclusions, where the static whole motion and the dynamic whole motion are dealt with, respectively.

Example 1 (static whole motion). Consider the hexagon formation of six agents in the two-dimensional space. The dynamics of each agent in swarm system (1) is given as follows:

$$
\begin{aligned}
& A=\left[\begin{array}{ll}
0 & I_{2} \\
0 & 0
\end{array}\right], \\
& B=\left[\begin{array}{l}
0 \\
I_{2}
\end{array}\right] .
\end{aligned}
$$
1 that

Set $Q=0.8 I_{4}$ and $\gamma=5$; then one can obtain by Theorem

$$
\begin{aligned}
K_{u} & =\left[\begin{array}{cccc}
1.8861 & 0 & 2.0304 & 0 \\
0 & 1.8861 & 0 & 2.0304
\end{array}\right], \\
K_{w} & =\left[\begin{array}{cccc}
3.5574 & 0 & 3.8295 & 0 \\
0 & 3.5574 & 0 & 3.8295 \\
3.8295 & 0 & 4.1225 & 0 \\
0 & 3.8295 & 0 & 4.1225
\end{array}\right] .
\end{aligned}
$$

And $J_{r}^{*}=320.42$. The communication network is presented in Figure 1, where the initial weights of all connected edges are one. The two-dimensional formation control vector $\iota=\left[\iota_{1}^{\mathrm{T}}, \iota_{2}^{\mathrm{T}}, \iota_{3}^{\mathrm{T}}, \iota_{4}^{\mathrm{T}}, \iota_{5}^{\mathrm{T}}, \iota_{6}^{\mathrm{T}}\right]^{\mathrm{T}}$ is given in Table 1 , where initial values of position-similar states and velocity-similar states are also shown. It can be found that the average value of initial states of velocity-similar states is equivalent to zero.

Figure 2 shows the state snapshots of six agents, and the whole motion is given at $J_{r}(t) \mathrm{s}, J_{r}(t) \mathrm{s}, J_{r}(t) \mathrm{s}$, and $J_{r}(t) \mathrm{s}$, where six agents are represented by asterisks, plusses, circles, $\mathrm{x}$-marks, pentagrams, and hexagrams, respectively, and the center point of the whole motion is denoted by red circles, which is determined by Theorem 3 . It can be found that all agents achieve a time-invariant hexagon formation and the center point of the whole motion is static. Figure 3 shows that the practical performance function $J_{r}(t)$ tends to a finite 


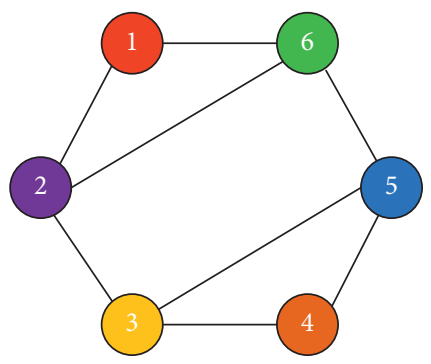

FIgure 1: Communication network for static whole motion.

TABLE 1: Initial values and formation vectors for static whole motion.

\begin{tabular}{lccc}
\hline Serial number & $\left(x_{i 1}(0), x_{i 2}(0)\right)$ & $\left(v_{i 1}(0), v_{i 2}(0)\right)$ & $\left(\iota_{i 1}, \iota_{i 2}\right)$ \\
\hline 1 & $(-5,-1)$ & $(-1,0.5)$ & $(2,0)$ \\
2 & $(-3,0)$ & $(-0.8,-1)$ & $(1,1.732)$ \\
3 & $(0,-1)$ & $(0.3,-0.2)$ & $(-1,1.732)$ \\
4 & $(3,3)$ & $(0.2,0.2)$ & $(-2,0)$ \\
5 & $(3,0)$ & $(-0.4,0.3)$ & $(-1,-1.732)$ \\
6 & $(2,-1)$ & $(0.1,0.2)$ & $(1,-1.732)$ \\
\hline
\end{tabular}
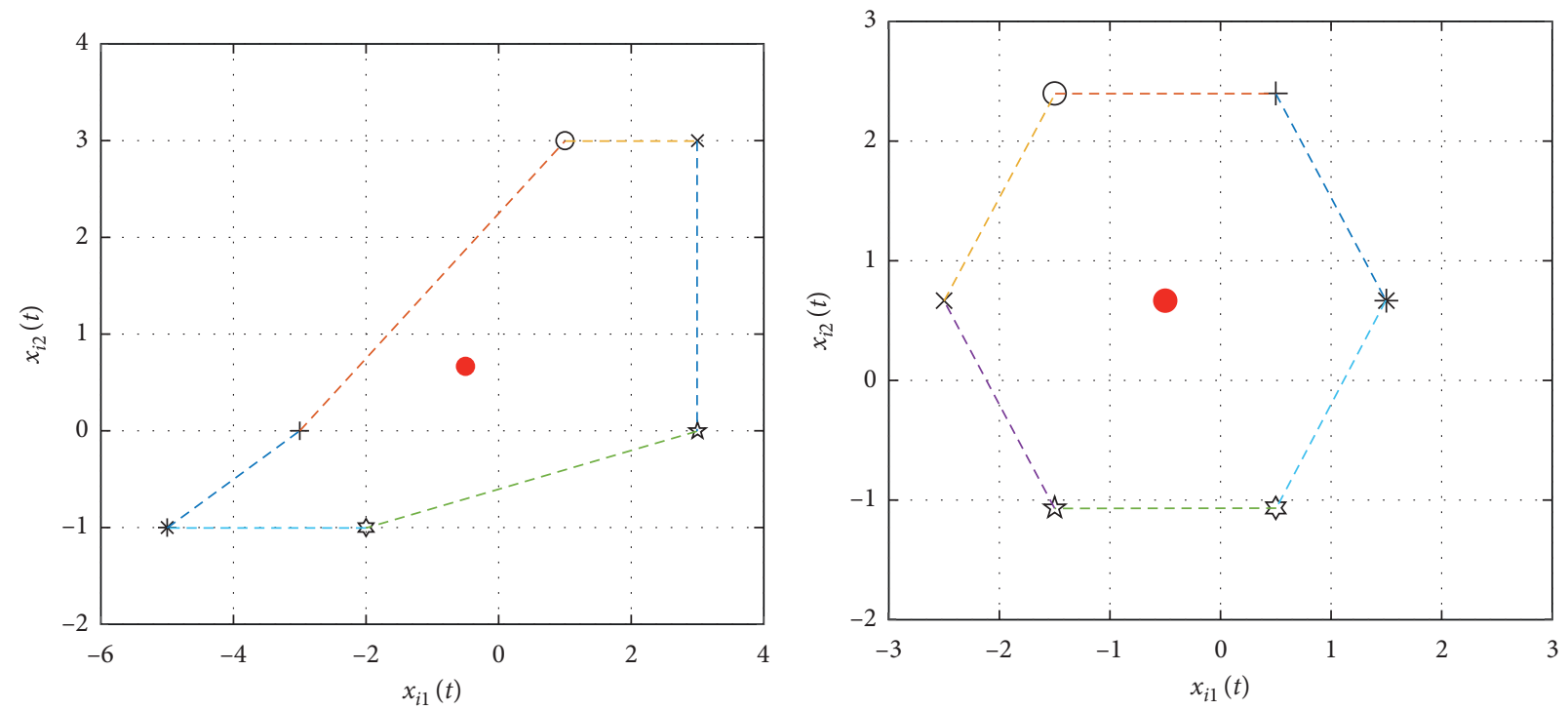

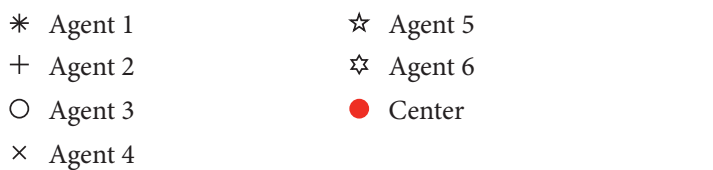

(a)

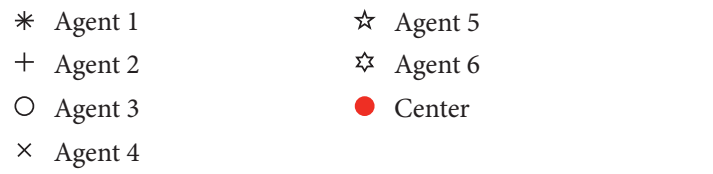

(b)

FIgUre 2: Continued. 


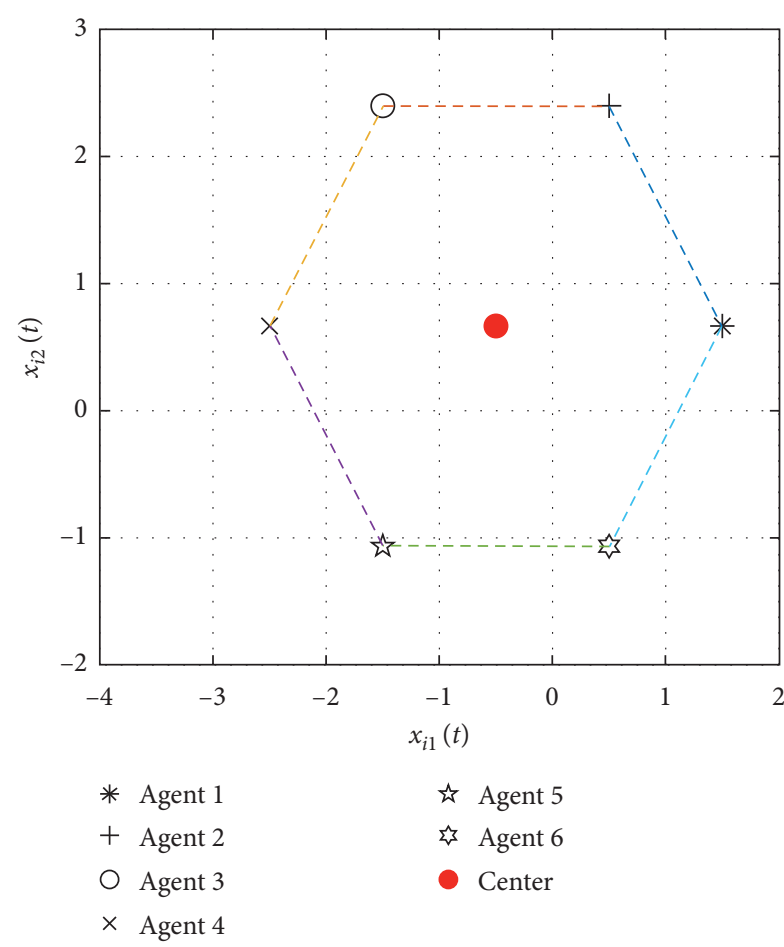

(c)

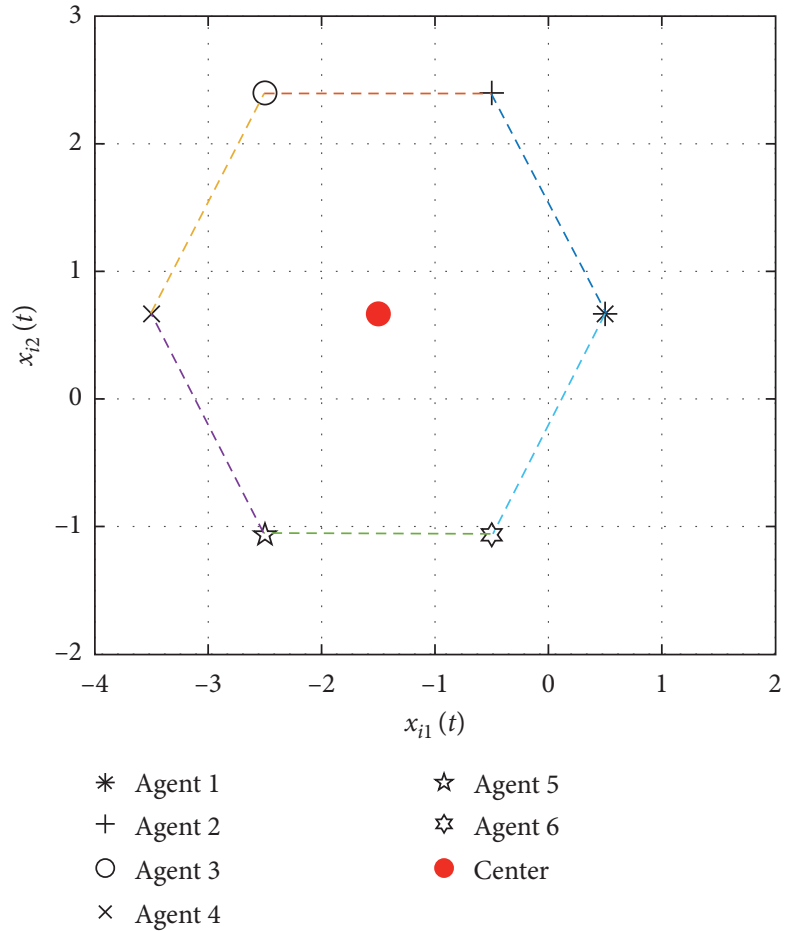

(d)

FIGURE 2: State snapshots for static whole motion. (a) $t=0 \mathrm{~s}$. (b) $t=14 \mathrm{~s}$. (c) $t=16 \mathrm{~s}$. (d) $t=18 \mathrm{~s}$.

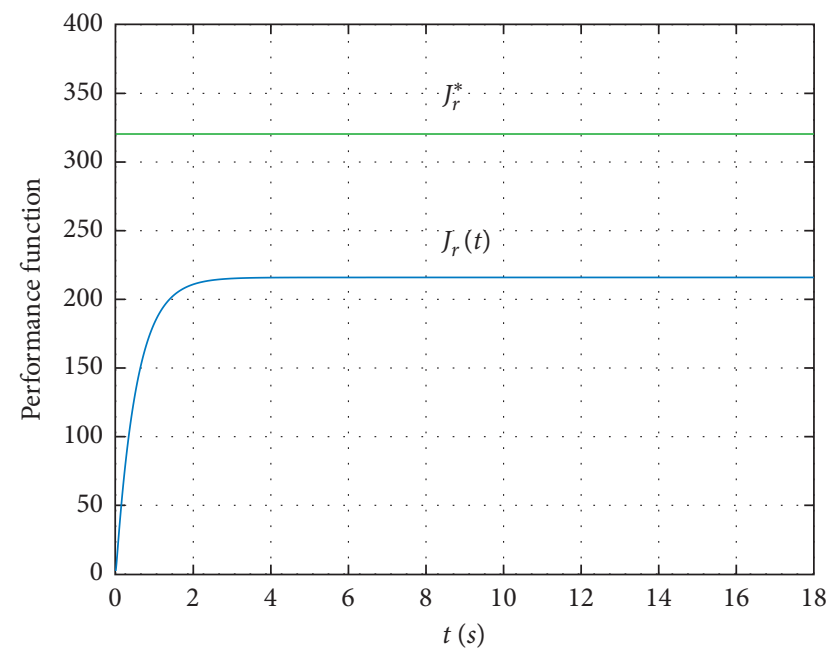

Figure 3: Performance function for static whole motion.

and stable value less than $J_{r}^{*}$, which is determined by Theorem 1. It can be found that the desired structure of this swarm system is achieved and the expected performance is guaranteed; that is, this swarm system achieves synchronization-based guaranteed-performance formation.

Example 2 (dynamic whole motion). Consider the pentagram formation structure of five agents in the three-dimensional space. In this case, each agent is modeled as

$$
\begin{aligned}
& A=\left[\begin{array}{ll}
0 & I_{3} \\
0 & 0
\end{array}\right], \\
& B=\left[\begin{array}{l}
0 \\
I_{3}
\end{array}\right] .
\end{aligned}
$$

Let $Q=I_{4}$ and $\gamma=3$; then gain matrices can be determined from Theorem 1 as

$$
\begin{aligned}
K_{u} & =\left[\begin{array}{cccccc}
2.6226 & 0 & 0 & 2.9452 & 0 & 0 \\
0 & 2.6226 & 0 & 0 & 2.9452 & 0 \\
0 & 0 & 2.6226 & 0 & 0 & 2.9452
\end{array}\right], \\
K_{w} & =\left[\begin{array}{cccccc}
6.8782 & 0 & 0 & 7.7243 & 0 & 0 \\
0 & 6.8782 & 0 & 0 & 7.7243 & 0 \\
0 & 0 & 6.8782 & 0 & 0 & 7.7243 \\
7.7243 & 0 & 0 & 8.6745 & 0 & 0 \\
0 & 7.7243 & 0 & 0 & 8.6745 & 0 \\
0 & 0 & 7.7243 & 0 & 0 & 8.6745
\end{array}\right] .
\end{aligned}
$$

And $J_{r}^{*}=1214.31$. The communication network with five agents is shown in Figure 4, where the initial weights of all connected edges are set as 1 , which is regulated according to formation protocol (5). Table 2 shows the three-dimensional formation control vector $\iota=\left[l_{1}^{\mathrm{T}}, l_{2}^{\mathrm{T}}, l_{3}^{\mathrm{T}}, l_{4}^{\mathrm{T}}, l_{5}^{\mathrm{T}}\right]^{\mathrm{T}}$ and initial values of position-similar states and velocity-similar states, where the average of initial values of velocity-similar states is nonzero. 


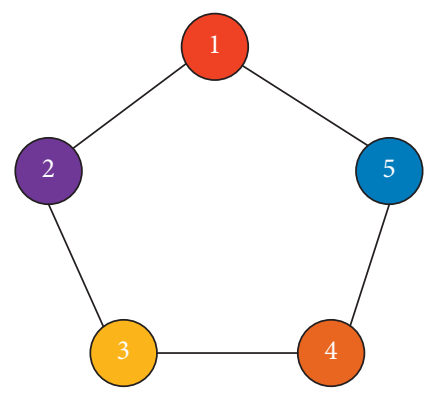

Figure 4: Communication network for dynamic whole motion.

TABLE 2: Initial values and formation vectors for dynamic whole motion.

\begin{tabular}{lccr}
\hline Serial number & $\left(x_{i 1}(0), x_{i 2}(0), x_{i 3}(0)\right)$ & $\left(v_{i 1}(0), v_{i 2}(0), v_{i 3}(0)\right)$ & $\left(\iota_{i 1}, \iota_{i 2}, \iota_{i 3}\right)$ \\
\hline 1 & $(-2.0,3.0,2.0)$ & $(1,0.5,0)$ & $(-10.4,0,0)$ \\
2 & $(2.0,4.5,2)$ & $(-2.0,2.0,1.5)$ & $(-3.2,5.7,5.7)$ \\
3 & $(-1.3,5.5,1.3)$ & $(1.5,-1.0,-3.0)$ & $(8.4,3.5,-3.5)$ \\
4 & $(-3.1,2.3,2.1)$ & $(3.0,0.8,1.0)$ & $(8.4,-3.5,3.5)$ \\
5 & $(-2.5,1.3,4.0)$ & $(-1.0,1.0,0)$ & $(-3.2,-5.7,5.7)$ \\
\hline
\end{tabular}

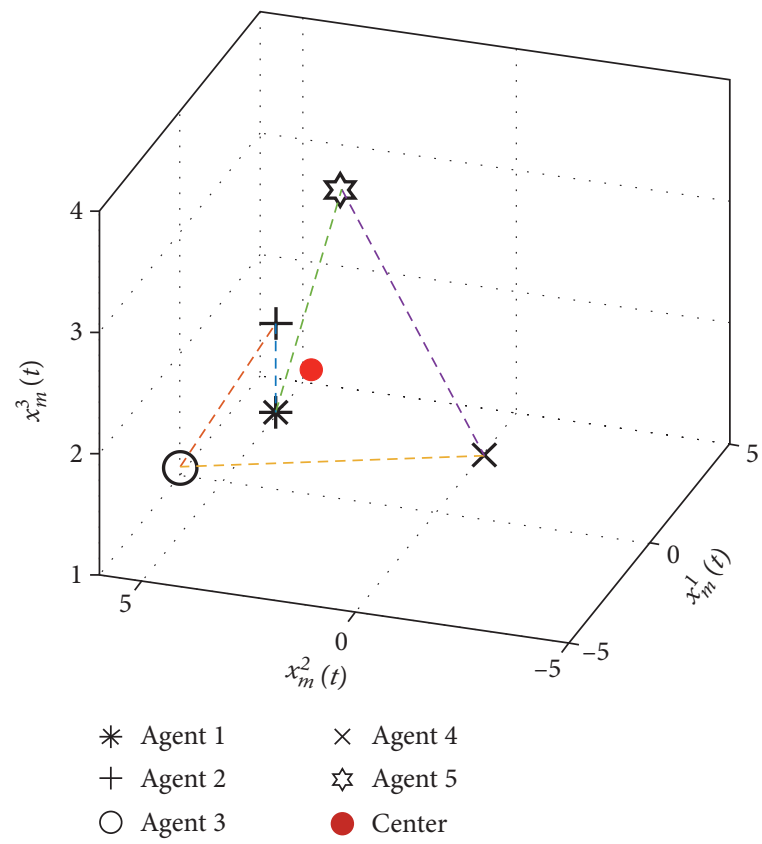

(a)

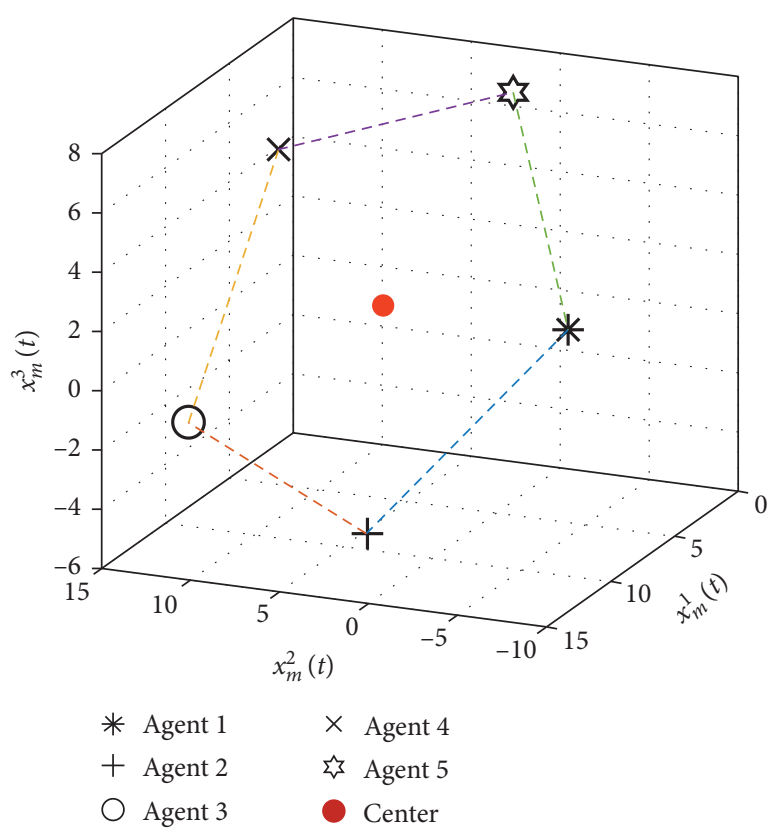

(b)

Figure 5: Continued. 


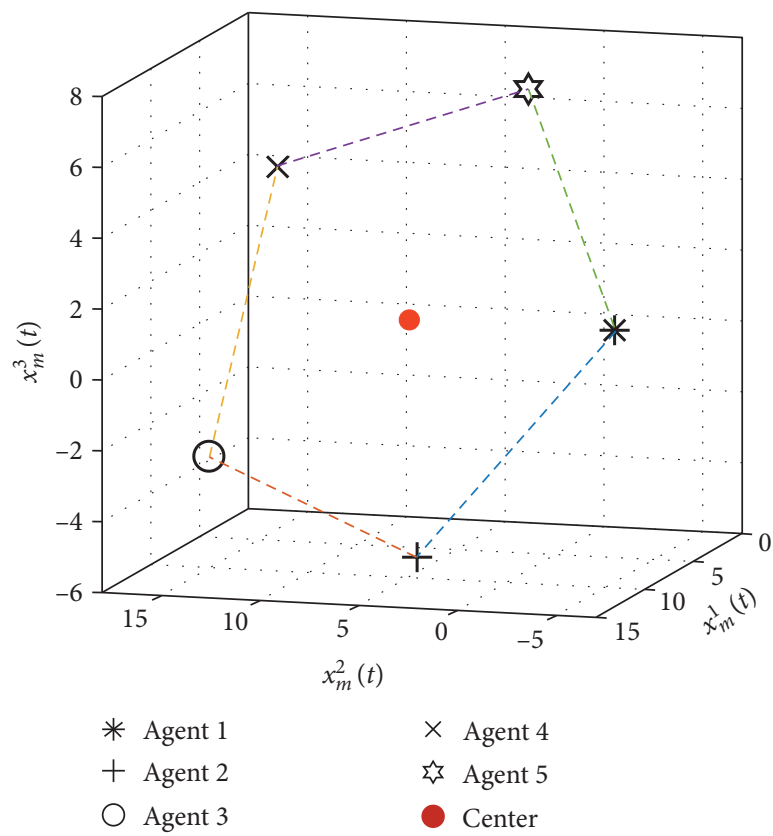

(c)

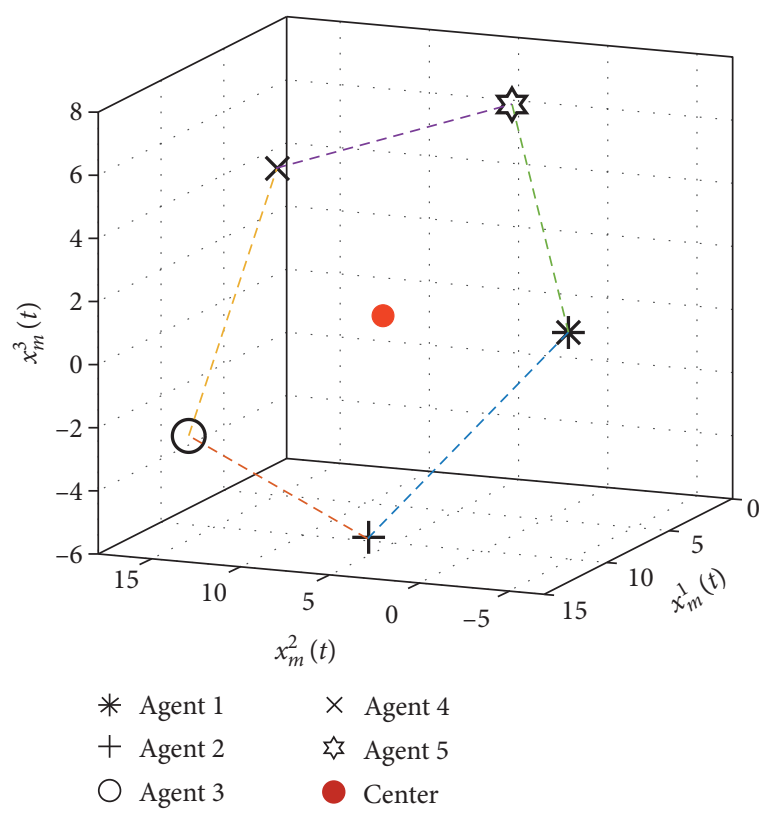

(d)

Figure 5: State snapshots for dynamic whole motion. (a) $t=0 \mathrm{~s}$. (b) $t=14 \mathrm{~s}$. (c) $t=16 \mathrm{~s}$. (d) $t=18 \mathrm{~s}$.

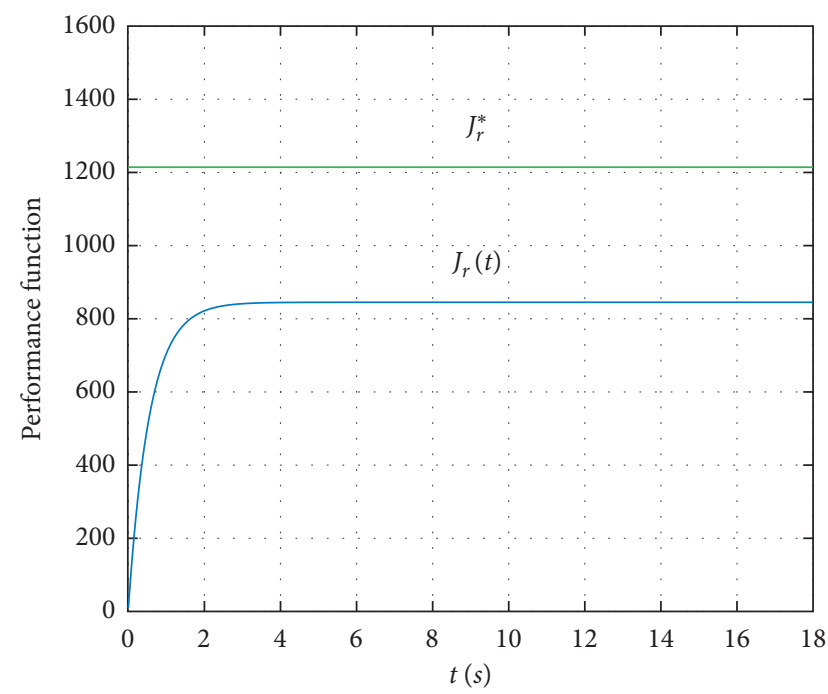

Figure 6: Performance function for dynamic whole motion.

In Figure 5, the state snapshots of five agents and the whole motion are revealed at $J_{r}(t) \mathrm{s}, J_{r}(t) \mathrm{s}, J_{r}(t) \mathrm{s}$, and $J_{r}(t)$ s, where five agents are denoted by asterisks, plusses, circles, $\mathrm{x}$-marks, and hexagrams, respectively, and the center point of the whole motion is denoted by red circles. One can see that all agents achieve a pentagram formation, where the pentagram is fixed, but the center point of the whole motion is time-varying and is consistent with Theorem 3. Figure 6 reveals the practical performance function $J_{r}(t)$ and the guaranteed-performance cost $J_{r}^{*}$, which is determined by Theorem 1. One can see that this swarm system achieves the desired synchronization-based guaranteed-performance formation.

\section{Conclusions}

Synchronization-based guaranteed-performance formation design problems for second-order swarm systems were addressed. A guaranteed-performance formation protocol was proposed, where the connected-edge weights are adaptively regulated on the basis of state errors and formation errors among neighboring agents. Furthermore, on the basis of the Riccati inequality, synchronization-based guaranteed-performance formation criteria were proposed, and an explicit expression of the guaranteed-performance cost was given. Those criteria are fully distributed in the sense that the gain matrix design of the formation protocol is independent of global information of swarm systems. Moreover, the whole motion of a swarm system was determined, where the impacts of position-similar states, velocity-similar states, and formation control vectors are shown, respectively. Particularly, it was shown that when the synchronizationbased guaranteed-performance formation is achieved, the formation center of the whole swarm system can be static and can also move in the uniform linear mode. Moreover, it is a very interesting future work to establish a unified 
criterion, which can compare similar methods associated with the guaranteed-performance formation control.

\section{Data Availability}

The simulation data used to support the findings of this study are included within the article.

\section{Conflicts of Interest}

The authors declare that they have no conflicts of interest regarding the publication of this paper.

\section{Authors' Contributions}

Hongtao Dang and Yichen Du conceptualized the study; Lingyun Kong was responsible for methodology; Hui Yao validated the study; Jianxiang $\mathrm{Xi}$ contributed to formal analysis; Hongtao Dang investigated the study; Yichen Du was responsible for writing and preparation of original draft; Hui Yao and Lingyun Kong were responsible for writing-review and editing; Jianxiang Xi supervised the study; Hongtao Dang was responsible for project administration; and Hongtao Dang acquired funding. All authors have read and agreed to the published version of the manuscript.

\section{Acknowledgments}

This research was funded by the Key Research and Development Program of Shaanxi (no. 2019GY-025).

\section{References}

[1] R. Olfati-Saber, "Flocking for multi-agent dynamic systems: algorithms and theory," IEEE Transactions on Automatic Control, vol. 51, no. 3, pp. 401-420, 2006.

[2] J. Zhou, X. Wu, W. Yu, M. Small, and J.-A. Lu, "Flocking of multi-agent dynamical systems based on pseudo-leader mechanism," Systems \& Control Letters, vol. 61, no. 1, pp. 195-202, 2012.

[3] J. Xi, C. Wang, H. Liu, and L. Wang, "Completely distributed guaranteed-performance consensualization for high-order multiagent systems with switching topologies," IEEE Transactions on Systems, Man, and Cybernetics: Systems, vol. 49, no. 7, pp. 1338-1348, 2019.

[4] J. Qu, Z. Ji, C. Lin, and H. Yu, "Fast consensus seeking on networks with antagonistic interactions," Complexity, vol. 2018, Article ID 7831317, 2018.

[5] J. Sun, Z. Geng, Z. Li, and Z. Ding, "Distributed adaptive consensus disturbance rejection for multi-agent systems on directed graphs," IEEE Transactions on Control of Network Systems, vol. 5, no. 1, pp. 202-212, 2018.

[6] J. Xi, C. Wang, X. Yang, and B. Yang, "Limited-budget output consensus for descriptor multiagent systems with energy constraints," IEEE Transactions on Cybernetics, pp. 1-13, 2020.

[7] Y. Zhang, H. Li, J. Sun, and W. He, "Cooperative adaptive event-triggered control for multi-agent systems with actuator failures," IEEE Transactions on Systems, Man and Cybernetics: Systems, vol. 49, no. 9, pp. 1759-1768, 2019.

[8] J. Xi, M. He, H. Liu, and J. Zheng, "Admissible output consensualization control for singular multi-agent systems with time delays," Journal of the Franklin Institute, vol. 353, no. 16, pp. 4074-4090, 2016.

[9] J. Xi, L. Wang, J. Zheng, and X. Yang, "Energy-constraint formation for multiagent systems with switching interaction topologies," IEEE Transactions on Circuits and Systems I: Regular Papers, vol. 67, no. 6, pp. 2442-2454, 2020.

[10] Z.-Y. Tan, N. Cai, J. Zhou, and S.-G. Zhang, "On performance of peer review for academic journals: analysis based on distributed parallel system," IEEE Access, vol. 7, pp. 19024-19032, 2019.

[11] L. Consolini, F. Morbidi, D. Prattichizzo, and M. Tosques, "Leader-follower formation control of nonholonomic mobile robots with input constraints," Automatica, vol. 44, no. 5, pp. 1343-1349, 2008.

[12] K.-K. Oh and H.-S. Ahn, "Formation control of mobile agents based on distributed position estimation," IEEE Transactions on Automatic Control, vol. 58, no. 3, pp. 737-742, 2013.

[13] H. Liu, T. Ma, F. L. Lewis, and Y. Wan, "Robust formation control for multiple quadrotors with nonlinearities and disturbances," IEEE Transactions on Cybernetics, vol. 50, no. 4, pp. 1362-1371, 2020.

[14] W. Ren, "Consensus strategies for cooperative control of vehicle formations," IET Control Theory \& Applications, vol. 1, no. 2, pp. 505-512, 2007.

[15] J. A. Fax and R. M. Murray, "Information flow and cooperative control of vehicle formations," IEEE Transactions on Automatic Control, vol. 49, no. 9, pp. 1465-1476, 2004.

[16] H. Du, G. Wen, Y. Cheng, Y. He, and R. Jia, "Distributed finite-time cooperative control of multiple high-order nonholonomic mobile robots," IEEE Transactions on Neural Networks and Learning Systems, vol. 28, no. 12, pp. 29983006, 2016.

[17] W. Qin, Z. Liu, and Z. Chen, “A novel observer-based formation for nonlinear multi-agent systems with time delay and intermittent communication," Nonlinear Dynamics, vol. 79, no. 3, pp. 1651-1664, 2015.

[18] L. Wang, J. Xi, M. He, and G. Liu, "Robust time-varying formation design for multiagent systems with disturbances: extended state observer method," International Journal of Robust and Nonlinear Control, vol. 30, no. 7, pp. 2796-2808, 2020.

[19] R. Rahimi, F. Abdollahi, and K. Naqshi, "Time-varying formation control of a collaborative heterogeneous multi agent system," Robotics and Autonomous Systems, vol. 62, no. 12, pp. 1799-1805, 2014.

[20] X. Dong and G. Hu, "Time-varying formation control for general linear multi-agent systems with switching directed topologies," Automatica, vol. 73, no. 73, pp. 47-55, 2016.

[21] D. I. Martinez, J. J. De Rubio, T. M. Vargas, V. Garcia, and G. Ochoa, "Stabilization of robots with a regulator containing the sigmoid mapping," IEEE Access, vol. 8, no. 1, pp. 89479-89488, 2020.

[22] J. Aguilar-Ibanez, G. Ochoa, D. Mujica-Vargas, E. Garcia, and R. Balcazar, "Structure regulator for the perturbations attenuation in a quadrotor," IEEE Access, vol. 7, no. 1, pp. 138244-138252, 2019.

[23] J. O. Escobedo-Alva, E. C. García-Estrada, L. A. PáramoCarranza, J. Meda Campana, and J. A. Meda-Campana, "Theoretical application of a hybrid observer on altitude tracking of quadrotor losing GPS signal," IEEE Access, vol. 6, pp. 76900-76908, 2018.

[24] J. D. J. Rubio, D. I. Martinez, V. Garcia, G. J. Gutierrez, and T. M. Vargas, "The perturbations estimation in two gas plants,” IEEE Access, vol. 8, no. 1, pp. 83081-83091, 2020. 
[25] C. Aguilar-Ibanez and M. S. Suarez-Castanon, "A trajectory planning based controller to regulate an uncertain 3D overhead crane system," International Journal of Applied Mathematics and Computer Science, vol. 29, no. 4, pp. 693702, 2019.

[26] J. H. Pérez-Cruz, P. A. Tamayo-Meza, M. Figueroa, and R. Silva-Ortigoza, "Exponential synchronization of chaotic xian system using linear feedback control," Complexity, vol. 2019, pp. 1-10, 2019.

[27] F. Xiao, L. Wang, J. Chen, and Y. Gao, "Finite-time formation control for multi-agent systems," Automatica, vol. 45, no. 11, pp. 2605-2611, 2009.

[28] X. W. Dong, Y. Zhou, Z. Ren, and Y. S. Zhong, "Time-varying formation tracking for second-order multi-agent systems subjected to switching topologies with application to quadrotor formation flying," IEEE Transactions on Industrial Electronics, vol. 64, no. 6, pp. 5014-5024, 2017.

[29] X. Dong and G. Hu, "Time-varying formation tracking for linear multiagent systems with multiple leaders," IEEE Transactions on Automatic Control, vol. 62, no. 7, pp. 36583664, 2017.

[30] J. Xi, Z. Fan, H. Liu, and T. Zheng, "Guaranteed-cost consensus for multiagent networks with Lipschitz nonlinear dynamics and switching topologies," International Journal of Robust and Nonlinear Control, vol. 28, no. 7, pp. 2841-2852, 2018.

[31] L. Wang, J. Xi, M. Yuan, and G. Liu, "Guaranteed-performance time-varying formation control for swarm systems subjected to communication constraints," IEEE Access, vol. 6, pp. 45384-45393, 2018.

[32] J. Yu, X. Dong, Q. Li, and Z. Ren, "Robust $H_{\infty}$ guaranteed cost time-varying formation tracking for high-order multiagent systems with time-varying delays," IEEE Transactions on Systems, Man and Cybernetics: Systems, vol. 50, no. 4, pp. 1465-1475, 2020.

[33] C. Godsil and G. Royal, Algebraic Graph Theory, SpringerVerlag, New York, NY, USA, 2001.

[34] S. Boyd, L. E. Ghaoui, E. Feron, and V. Balakrishnan, Linear Matrix Inequalities in System and Control Theory, SIAM, Philadelphia, PA, USA, 1994.

[35] Z. Li, G. Wen, Z. Duan, and W. Ren, "Designing fully distributed consensus protocols for linear multi-agent systems with directed graphs," IEEE Transactions on Automatic Control, vol. 60, no. 4, pp. 1152-1157, 2015. 